\title{
Using the theory of planned behavior to identify predictors of handwashing among Iranian healthcare workers
}

\author{
M Askarian ${ }^{1 *}$, N Maharlouei $^{2}$, F Yousefi $^{2}$, M-L Mclaws ${ }^{3}$ \\ From International Conference on Prevention \& Infection Control (ICPIC 2011) \\ Geneva, Switzerland. 29 June - 2 July 2011
}

\section{Introduction / objectives}

The incidence of healthcare associated infections in a surgical ward in Shiraz has been $\hat{A}$ established as $18 \%$ $\hat{A}$ with associated costs of approx US150,000. Healthcare workers (HCWs) had acceptable levels of knowledge and attitude about hand hygiene but poor self-reported practices. We used the Theory of Planned Behavior (TPB) to identify predictors of handwashing to underpin a theory-driven intervention.

\section{Methods}

Between April and September 2008, 1700 healthcare workers (HCWs) from all wards in 18 private and 10 public hospitals in Shiraz answered a self-administered survey designed in accordance with the TPB. Multiple logistic regression analysis was used to model two handwashing for patient contacts perceived to be clean and contacts perceived to contaminate hands.

\section{Results}

90\% of HCWs returned a completed survey. Significant $\hat{A}$ predictors for clean contact handwashing in the hospital included compliance with $\hat{A}$ similar community practice (AOR2.1, $\mathrm{P}<0.000)$ and contaminated contact handwashing $\hat{\mathrm{A}}$ (AOR1.6, $\mathrm{P}<0.000$ ), perception that clean contact handwashing $\hat{A}$ required little effort (AOR1.1, $\mathrm{P}=0.039$ ) and nursing peer pressure (AOR $1.1, \mathrm{P}=0.025)$. Significant $\hat{A}$ predictors for contaminated contact handwashing included clean contact handwashing compliance (AOR2.5, $\mathrm{P}<0.000)$, community contaminated contact handwashing (AOR1.5 $\mathrm{P}=0.001$ ), peer pressure from ICPs (AOR1.4, $\mathrm{P}=0.001)$ and attitudes about contaminated contact handwashing Â (AOR1.1, P=0.001).

\section{Conclusion}

Community-based handwashing practices exert strong influence on handwashing in the hospital. Given the interdependence between community and hospital handwashing a campaign to improve aware about the benefit of community handwashing may improve HCWs' compliance.

\section{Disclosure of interest}

None declared.

\section{Author details}

${ }^{1}$ Community Medicine, Shiraz University of Medical Sciences, Shiraz, Iran, Islamic Republic Of. ${ }^{2}$ Shiraz University, Shiraz, Iran, Islamic Republic Of.

${ }^{3}$ Public Health \& Community Medicine, UNSW, Sydney, Australia.

Published: 29 June 2011

\section{doi:10.1186/1753-6561-5-S6-P108}

Cite this article as: Askarian et al:: Using the theory of planned behavior to identify predictors of handwashing among Iranian healthcare workers. BMC Proceedings 2011 5(Suppl 6):P108. 